\title{
Exploring the Impact of Mandatory Remote Work during the COVID-19 Pandemic
}

\author{
Talha Oz ${ }^{1,2[0000-0002-6797-2010]}$ and Andrew Crooks ${ }^{1,3[0000-0002-5034-6654]}$ \\ ${ }^{1}$ George Mason University, Fairfax VA 22030, USA \\ ${ }^{2}$ Humanyze, Menlo Park CA, USA \\ ${ }^{3}$ University at Buffalo, Buffalo NY 14261, USA \\ toz@gmu.edu atcrooks@buffalo.edu
}

\begin{abstract}
During the early months of the COVID-19 pandemic, millions of people had to work from home. We examine the ways in which COVID-19 affect organizational communication by analyzing five months of calendar and messaging metadata from a technology company. We found that: (i) cross-level communication increased more than that of same-level, (ii) while within-team messaging increased considerably, meetings stayed the same, (iii) off-hours messaging became much more frequent, and that this effect was stronger for women; (iv) employees respond to non-managers faster than managers; finally, (v) the number of short meetings increased while long meetings decreased. These findings contribute to theories on organizational communication, remote work, management, and flexibility stigma. Besides, this study exemplifies a strategy to measure organizational health using an objective (not self-report based) method. To the best of our knowledge, this is the first study using workplace communication metadata to examine the heterogeneous effects of mandatory remote work.
\end{abstract}

Keywords: Work from Home, Communication, COVID-19, Organization.

\section{$1 \quad$ Introduction}

The rapidly evolving COVID-19 crisis challenged millions of employees all around the world to start working from home (WFH) almost overnight, unprepared. More than one third of the U.S. workforce that used to commute before March 2020 were sent to their homes to WFH until a further notice [1]. Accordingly, employers have been actively looking for WFH policies which promote effective WFH and limit the impact of the pandemic. In order to do this, they first need to identify how WFH affect their employees' communication and collaboration behavior. Without knowing it, millions of employees' health and organizations' productivity are at great risk. Because, now the workforce has to deal not only with the traditional stressors of WFH, but also with the pandemic, which might be the most extreme global stressor of our generation [2].

After shortly experiencing WFH during COVID-19, many big tech firms including Facebook and Twitter announced that they are transitioning into being "remote-first" 
[3]. Research not only shows how disasters and pandemics have immediate effects on organizational behavior [4-6], but it also identified how early responses to chaotic events centuries ago effect companies' behaviors even today [7]. Accordingly, all employers need to identify the most effective WFH policies for their companies urgently.

\section{Hypothesis Development}

When employees don't work in business as usual (BAU) settings but are forced to WFH -due to disasters or pandemics- they have to compensate for the lost opportunity of face-to-face (f2f) communication. While some of these communications happen in planned meetings, others happen impromptu. Although there is a high variation in the findings of prior studies on the amount of scheduled meetings [8] and unscheduled $\mathrm{f} 2 \mathrm{f}$ communication $[9,10]$, they report that at least $25 \%$ of work time is spent for scheduled meetings and another $25 \%$ for unscheduled communication during normal times. After transitioning to WFH, we can assume for regular planned meetings would continue in a virtual setting. The fate of the unplanned $\mathrm{f} 2 \mathrm{f}$ communication is more complicated when it comes to WFH.

Communications for coordinating social activities such as having a meal or drink together is no longer relevant and hence cease during WFH. Others move to available computer mediated communication channels such as virtual meetings (e.g. Zoom), email, and workplace messaging apps (WMA) such as Slack and Microsoft Teams.

During WFH we expect (H1) much of the unplanned $f 2 f$ communication to move to $W M A$. Our rationale is that many functions of WMA are similar to $\mathrm{f} 2 \mathrm{f}$ (and if not better). In the sense, it allows for: (i) negotiating availability which gives more control to recipient and solves the convenience asymmetry problem of f2f interruptions while (ii) maintaining social connections and conversational context (e.g., emojis and persistent chat history); (iii) furthermore, quick questions and clarifications via instant messaging is faster than dialing in, and, (iv) WMA allows for context-rich interactions by utilizing topic-based groups and message-threads as well as (v) coordination and scheduling with dyadic/multi-party direct messages and integrated apps/bots $[10,11]$.

Despite the many benefits of WMA, Nardi et al. [11] observed that employees switch media when the matter discussed is complicated, when there is a misunderstanding, or when communicating information requires too much typing. In this regard, media richness theory [12] suggests that complex information is better transmitted in rich channels where it is easier to ask questions, interrupt, interpret cues, and give feedback. Accordingly, we expect ( $\mathrm{H} 2$ ) employees to setup extra (short) meetings when WFH.

Often, organizations are divided into divisions and further into teams. The members of a team depend upon and communicate among each other more than others. Therefore, (H3) we expect messaging and meeting dyads within teams to increase more than those of cross-teams. Besides, research on the effects of reorganization on tie decay suggests that employees are more likely to choose to retain ties to valuable contacts, reciprocated ties, and socially embedded ties [13], and we assume that employees are embedded in their teams and have higher reciprocity with their team members. 
Supervisors should support their employees because it has been found that supervisor support is positively related to job satisfaction [14], in particular when employees are WFH [15]. Another benefit of greater leader-member information exchange is that subordinates then show more helping behavior to their coworkers [16]. From a Machiavellian perspective, managers are among the most valuable contacts with whom to retain ties [13]. Therefore, we expect that $(\mathrm{H} 4)$ cross-level messaging increases more than that of same-level when WFH becomes mandatory.

Teleworking provides flexibility and autonomy, and this has been shown to be highly appreciated by employees WFH during disasters and pandemics, e.g., $[4,6]$. Due to the closures of facilities and schools, family role demands and interference with work greatly increased in the early months of COVID-19. Thus, work has become more fragmented and sporadic throughout the day. Therefore, we expect (H5) employees to work more and communicate more outside regular business hours. Furthermore, it has been shown that household labor is not shared equally in most families. Working mothers assume a much greater responsibility for household management and child adjustment $(\sim 22 \mathrm{~h}$ vs $\sim 10 \mathrm{~h}$ per week for fathers) [17]. In another study the majority of working mothers with dependent children at home reported that "they alone assumed responsibility for household routines involving organizing schedules for the family and maintaining order in the home" [18]. Since many schools and childcare facilities were closed during the early months of the pandemic, and women often take on more household and childcare responsibilities during the day, their work might be more sporadic than that of men. Besides, women are more likely to suffer from the stigma against flexible workers [19], and therefore we expect (H6) women to be more willing to signal that they are working in the after-hours as an attempt to prevent that discrimination.

Work telepressure, the urge to respond to asynchronous work messages quickly, is found to be associated with the amount of work performed at home [20]. Since employees are WFH all of the time during COVID-19, $(H 7)$ we expect greater level of telepressure and hence quicker message turnaround, (H8) especially those who used to share the same office and had the opportunity to communicate $f 2 f$. Besides, researchers have noted a flexibility stigma by managers against those who WFH every day as not being as productive or as committed to those who come to the workplace [19]. In order to overcome the stigma while WFH, we expect (H9) employees to respond to messages of their managers even quicker.

Turning to meetings, we expect that (H10) employees reduce long meetings when $W F H$. Our rationale for this is that too many meetings or communication may cause difficulties for employees to find time to focus and do the actual work. Furthermore, it has been found that employees become annoyed when they are interrupted by meetings (rather than informal $\mathrm{f} 2 \mathrm{f}$ interruptions) [21]. Besides, interruptions by chat messages has been shown to be much less disruptive than $\mathrm{f} 2 \mathrm{f}$ because the recipient has more control over the situation, the norms are different -social costs associated with not responding in $\mathrm{f} 2 \mathrm{f}$ is much higher, and the recipient can peek a message to get a sense of its urgency to decide when to respond -such plausible deniability is not possible in $\mathrm{f} 2 \mathrm{f}$ or calls [11]. Also, meetings being too lengthy is one of the top reported meeting problems [8]; and, attending virtual meetings for a long period is worse than that of physical meetings -aka Zoom fatigue [22, 23]. 


\section{$3 \quad$ Methodology and Data}

This study exploits metadata (no content) emitted from commonplace workplace technologies such as calendar, and workplace messaging apps collected from a tech company starting from 2020-01-06 to 2020-05-31. The company has two main offices, one $(\mathrm{N}=26)$ is located in the Eastern Time Zone $(E T)$, and the other $(\mathrm{N}=9)$ is in the Pacific Time Zone (PT). The PT office transitioned to WFH on 2020-03-09 and the ET office on 2020-03-16. Chats (WMA) and meetings (calendar events) before and after these dates are designated as pre (BAU) and post (WFH) - the company does not use email for internal communication practically, therefore, we excluded it in this analysis.

From the company's calendar system, we collected time, duration, and attendees list of every event setup; and from their WMA, we collected time, sender, and receivers list of every message sent during the entire period. Besides the communication metadata, we obtained the following participant demographics: office location, primary-team and division, is-manager, tenure, and gender. In extracting metadata, all of the personal identifiers were hashed on the client's servers. From this communication metadata, we created 21 weekly snapshots of meeting and messaging networks and analyzed them.

Each event contributes to a directed weighted network through a concept called "attention time" [24]. An event generates one or more directed weighted links that can be represented as a 3-tuple of (attention giver, attention receiver, attention minutes). For example, when A sends a message to B, then the tie (A, B, estimated message writing time) is created but no tie is created from $B$ to $A$. When A sends a message to $\mathrm{N}$ recipients, a tie is created between $\mathrm{A}$ and each recipient (i.e., $\mathrm{N}$ ties in total), each with a weight of "estimated message writing time" divided to N. We estimated message writing time rather than using pure message counts in this analysis because people have different styles of using these systems. Especially in instant messaging, employee A might prefer "hitting enter" more often and creating a separate message event for each sentence (or even for each word!), while employee B might choose sending less messages with longer content. When a person sends multiple messages quickly one after another, we cap the writing time of the subsequent messages by the previous messagesent, otherwise we cap message writing time at 5 minutes based on self-reported WMA usage data. When calculating response times, we only considered messages explicitly responding a particular message in threads.

For meetings we do not have a sender-receiver concept (unlike messages), they only have co-attendees, therefore attention is considered bi-directional. When $\mathrm{A}$ has a 30 minute one-on-one meeting with participant $\mathrm{B}$, both ties of $(\mathrm{A}, \mathrm{B}, 30)$ and $(\mathrm{B}, \mathrm{A}, 30)$ are created; similarly, a 30 minute meeting of three participants $(A, B, C)$ creates six links (the six permutations of a three-element set) each with 15 minute weights (attention times) - namely, (A,B,15), (A,C,15), (B,A,15), (B,C,15), (C,A,15), (C,B,15).

\section{$4 \quad$ Results}

We hypothesized in $\mathrm{H} 1$ that people who previously had the opportunity to communicate f2 f would message each other more while WFH. To operationalize H1, we compare the 
increase in messaging among employees who previously shared the same office to those did not. As a baseline, during BAU, we observed that $80.5 \%$ of communication happened within the office. After transitioning to WFH, as seen in Table 1, the increase in WMA within the same office coworkers is much greater than between different offices. While the WMA increase for the same office is statistically significant $(p<0.001)$, it is not the case for between different offices. These findings support H1.

Table 1. Change in communication with same/different office co-workers (person-week).

\begin{tabular}{l|llll} 
& $B A U(\mathrm{~min})$ & WFH (min) & Change (min) & Change ratio \\
\hline Same office WMA & 208.19 & 257.48 & +49.28 & $+23.67 \%$ \\
Other office WMA & 50.37 & 53.93 & +3.56 & $+7.07 \%$ \\
Same office Meeting & 186.64 & 222.78 & +36.14 & $+19.37 \%$ \\
Other office Meeting & 50.67 & 82.90 & +32.23 & $+63.60 \%$
\end{tabular}

We expected that after transitioning to WFH, the number of short meetings would increase (H2) while the number of long meetings would decrease (H10). In Table 1 we see that the overall time spent in meetings increased. Regarding meeting lengths, our first observation is that most of the meetings were already short in the pre (BAU) condition, and there were only a few long meetings per person-week. But despite that, in the WFH situation we still see an increase in the short meetings and decrease in long meetings (the change is evident in the ratio column of Table 2). Therefore, we find strong evidence in favor of $\mathrm{H} 2$ and $\mathrm{H} 10$.

Table 2. Meeting counts per duration category.

\begin{tabular}{l|llll} 
& Pre $($ BAU) & Post $($ WFH $)$ & Change (count) & Ratio (\%) \\
\hline$<30$ mins & 4.64 & 5.83 & 1.19 & $+25.53 \%$ \\
30 mins & 12.70 & 17.31 & 4.62 & $+36.35 \%$ \\
31-45 mins & 1.11 & 1.76 & 0.64 & $+58.01 \%$ \\
$46-60$ mins & 9.76 & 15.44 & 5.67 & $+58.11 \%$ \\
$1-1.5$ hours & 1.49 & 1.26 & -0.23 & $-15.21 \%$ \\
$1.5-2$ hours & 0.53 & 0.32 & -0.21 & $-40.23 \%$ \\
2-24 hours & 1.85 & 0.76 & -1.09 & $-59.02 \%$
\end{tabular}

Turning to H3, we hypothesized that both messaging and meeting within teams would increase more than that of between teams. In BAU, we observe that most (67.7\%) of the messaging happens within teams. After transitioning to $\mathrm{WFH}$, there is a greater increase in same-team communication compared to other-team as shown in Table 3. These findings support the messaging part of H3. However, number of meetings stays nearly the same within teams while it increases more than one hour per person-week across teams. This can be explained by the post-COVID employee engagement policies of the company such as happy hours, (more frequent) town halls, and leadership office hours into which everyone was invited and encouraged to join. 
Table 3. Change in communication with same/different team coworkers.

\begin{tabular}{l|llll} 
& BAU $(\mathrm{min})$ & WFH $(\mathrm{min})$ & Change $(\mathrm{min})$ & Change ratio \\
\hline Same-team WMA & 197.57 & 245.78 & +48.21 & $+24.40 \%$ \\
Other-team WMA & 94.28 & 100.09 & +5.81 & $+6.17 \%$ \\
Same-team Meeting & 212.41 & 219.19 & +6.78 & $+3.19 \%$ \\
Other-team Meeting & 96.03 & 175.57 & +79.54 & $+82.83 \%$
\end{tabular}

With respect to $\mathrm{H} 4$, we hypothesized that cross-level messaging and meeting increases more than same-level communication during WFH. Through our analysis we found the increase in messaging to be higher among cross level communication (31.69\%), and lower for same level communication $(10.91 \%)$. These findings support the first half of H4. We also hypothesized that an average manager increases their meetings with nonmanagers more than they increase their meetings with other managers. Similarly, we expected an average non-manager to increase their meetings with managers more than they would increase their meetings with other non-managers. The results are shown in Table 4. During BAU, we see that most of the meetings were among managers (an average manager spends $\sim 3$ hours/week meeting with other managers). This continues in WFH. However, managers started to have more meetings with non-managers so much so that an average manager's meeting time surpasses all other categories (by jumping from 110 minutes/week to 187 minutes/week). Just as managers increased their meetings with non-managers more than they did with other managers $(69.76 \%>$ $29.31 \%$ ), non-mangers increased their meetings with managers more than they did with other non-managers $(18.91 \%>11.62 \%)$. These findings support the latter half of $\mathrm{H} 4$.

Table 4. Same-/cross-level communication breakdown during BAU and WFH.

\begin{tabular}{l|lllll}
$\begin{array}{l}\text { From (is } \\
\text { manager?) }\end{array}$ & $\begin{array}{l}\text { To (is } \\
\text { manager?) }\end{array}$ & $\begin{array}{l}\text { Pre (BAU) } \\
\text { (minute) }\end{array}$ & $\begin{array}{l}\text { Post (WFH) } \\
\text { (minute) }\end{array}$ & $\begin{array}{l}\text { Change } \\
\text { (minute) }\end{array}$ & $\begin{array}{l}\text { Change } \\
\text { (Ratio) }\end{array}$ \\
\hline $\mathrm{N}$ & $\mathrm{N}$ & 153.93 & 179.76 & 25.83 & $16.78 \%$ \\
$\mathrm{~N}$ & $\mathrm{Y}$ & 88.02 & 114.25 & 26.23 & $29.80 \%$ \\
$\mathrm{Y}$ & $\mathrm{N}$ & 136.04 & 181.77 & 45.73 & $33.61 \%$ \\
$\mathrm{Y}$ & $\mathrm{Y}$ & 234.22 & 245.60 & 11.37 & $4.86 \%$ \\
Is-mngr? & Meet w/ & Pre (min) & Post (min) & Change & Ratio (\%) \\
$\mathrm{N}$ & $\mathrm{N}$ & 117.39 & 131.03 & 13.64 & $11.62 \%$ \\
$\mathrm{~N}$ & $\mathrm{Y}$ & 122.23 & 145.33 & 23.11 & $18.91 \%$ \\
$\mathrm{Y}$ & $\mathrm{N}$ & 110.27 & 187.19 & 76.92 & $69.76 \%$ \\
$\mathrm{Y}$ & $\mathrm{Y}$ & 184.91 & 239.10 & 54.19 & $29.31 \%$
\end{tabular}

We hypothesized that off-hours asynchronous communication is greater when WFH than during BAU (H5), and that this increase is greater for women (H6). To explore this, we calculated the number of WMA chat minutes outside normal business hours (9am-5pm employee's local time) for each workday. Table 5 shows the results of this analysis. Specifically, women had about the same amount of chat in BAU to men, but this was tripled when WFH, while for men chat only doubled. These findings support hypotheses $\mathrm{H} 5$ and $\mathrm{H} 6$. 
Table 5. Change in off-hours chat for men and women from BAU to WFH.

\begin{tabular}{lllll} 
Gender & $\begin{array}{l}\text { Pre }(\text { BAU) } \\
\text { (minutes) }\end{array}$ & $\begin{array}{l}\text { Post }(\text { WFH }) \\
\text { (minutes) }\end{array}$ & $\begin{array}{l}\text { Change } \\
\text { (minutes) }\end{array}$ & Ratio \\
\hline$F$ & 10.17 & 31.34 & +21.17 & $208.26 \%$ \\
$M$ & 11.66 & 21.04 & +9.37 & $80.38 \%$
\end{tabular}

We hypothesized that employees respond to their peers' messages faster when WFH (H7) and that this effect is stronger for same office workers (H8) and for managers (H9). To operationalize these hypotheses, for each reply message, we calculated message response time (delta). We included responses sent within 8 hours of original message and excluded others. Overall, we found that the median response time dropped by $18.65 \%$, supporting H7. However, when we break it down, we see that while employees' response speed to same office workers increased by $23.45 \%$, their responses to other office workers slowed down by $16.88 \%$ (Table 6). Median response time from non-managers to managers were not faster than that of between non-managers, so we did not find any evidence in favor of $\mathrm{H} 9$.

Table 6. Chat message response time statistics.

\begin{tabular}{l|llll}
\multicolumn{1}{c}{} & Pre (min) & Post (min) & Change (min) & Ratio \\
\hline Overall & 18.63 & 15.16 & -3.47 & $-18.65 \%$ \\
Other office & 23.80 & 27.82 & 4.02 & $+16.88 \%$ \\
Same office & 17.20 & 13.17 & -4.03 & $-23.45 \%$ \\
From-To (is manager) & & & & \\
$N-N$ & 17.73 & 12.93 & -4.80 & $-27.07 \%$ \\
$N-Y$ & 20.48 & 17.29 & -3.18 & $-15.55 \%$ \\
$Y-N$ & 16.59 & 14.05 & -2.54 & $-15.32 \%$ \\
$Y-Y$ & 19.73 & 19.11 & -0.63 & $-3.17 \%$
\end{tabular}

\section{Conclusion}

F2 $\mathrm{f}$ communication offer values that are not available in mediated communication, and lack of it brings risks. Beyond offering a richer medium for information exchange, sharing a common space provides opportunities for sustaining social relationships (through touching, eating/drinking, and informal interactions) which is a precondition for effective communication. It eases communication by allowing referencing to items, using pronouns, and using physical items in collaboration, and learning (by observing and maybe even subconsciously) [21, 25]. When WFH however, out of sight, out of mind attitude might win over, and hence information undersupply, role ambiguity, social isolation, disengagement, job dissatisfaction, and stress might increase [26]. Most of the hypotheses tested in this paper are adaptations to prevent such complications.

Employee stress is negatively associated with organizational financial success, team productivity, and employee engagement [27]. To mitigate their effects, employers try 
to identify work stressors (i.e. conditions causing stress in their organization). Identification of work stressors has traditionally been through surveys; however, Oz [28] argued that digital trails of commonplace workplace technologies can be exploited for this purpose and that this strategy has many advantages over the self-report based method. In this study, we extend that strategy to show the impact of COVID-19 on work practices using data collected from a technology company before and after it transitioned to WFH. This study contributes to the literature in several unique ways.

First, what we know of work practices during the COVID-19 is very limited despite more than one third of the U.S. workforce transitioned to WFH during the pandemic [1] -and many believe that WFH is here to stay [5]. Yet, empirical studies on the topic is very rare $[4,6,29,30]$. Accordingly, our first contribution is providing insights on a phenomenon that has become very prevalent but very less known.

Second contribution of this study is that it employs ICT (information and communication technologies) data while most prior studies were self-report based, and therefore suffer from well-known survey issues such as selectivity biases, response biases, and item-characteristic biases [31]. The only other work that uses ICT metadata for analyzing the effect of mandatory WFH is [29]. Although that study is sound from an external validity perspective, their data is at metropolitan area level and did not give itself in to analyze any heterogeneity effect of WFH on communication patterns. Therefore, their data did not give in much room for theory development and hypothesis testing.

Third, the surveys in the aforementioned studies were all conducted after employees transitioned to m-WFH. For example, even though the respondents were asked to selfassess their wellbeing for four weeks prior to beginning $\mathrm{m}-\mathrm{WFH}$ in [6], their responses might suffer from memory weaknesses, transient mood effects, and other biases. However, that is not the case with ICT data: it gives itself in for retrospective data collection, and thus solve the unobservability problem [32].

Fourth, most prior survey-based studies measure higher level constructs. For example, [6] examines responses to "My team is continuing to have regular meetings" and "My team is avoiding synchronous communication". Similarly, [4] ask questions such as "WFH lead to loss of professional/social interaction" and "WFH enabled me to have more time for myself/my family". We argue, by using atomic enacted events as constructs, one can learn more on the extent of such behaviors. Thus, this approach can help solve some of the paradoxes in the telework literature [33, 34].

Fifth, we develop hypotheses based on communication theories, in particular the theories of mediated communication [25] and telework [26]. Thus, this study is not a "theory free" big data analysis [35], but a "theory centric" computational social science study [36]. In this regard, this study is unlike the articles merely reporting statistics on the change of ICT use during COVID-19 and lack theoretical discussions [29, 37, 38].

\section{References}

1. Brynjolfsson, E., Horton, J., Ozimek, A., Rock, D., Sharma, G., Ye, H.Y.T.: Covid-19 and remote work: An early look at us data. Unpubl. Work. (2020). 
2. Sinclair, R.R., Allen, T., Barber, L., Bergman, M., Britt, T., Butler, A., Ford, M., Hammer, L., Kath, L., Probst, T., Yuan, Z.: Occupational Health Science in the Time of COVID-19: Now more than Ever. Occup. Health Sci. 1-22 (2020)..

3. Levy, S.: Remote-First Companies Are Another Covid-19 Calamity, wired.com/story/plaintext-remote-first-companies-are-another-covid-19-calamity/, (2020).

4. Donnelly, N., Proctor-Thomson, S.B.: Disrupted work: home-based teleworking (HbTW) in the aftermath of a natural disaster. New Technol. Work Employ. 30, 47-61 (2015).

5. Baert, S., Lippens, L., Moens, E., Weytjens, J., Sterkens, P.: The Covid-19 Crisis and Telework: A Research Survey on Experiences, Expectations and Hopes. Social Science Research Network, Rochester, NY (2020).

6. Ralph, P., Baltes, S., Adisaputri, G., Torkar, R., Kovalenko, V., Kalinowski, M., Novielli, N., Yoo, S., Devroey, X., Tan, X., Zhou, M., Turhan, B., Hoda, R., Hata, H., Robles, G., Fard, A.M., Alkadhi, R.: Pandemic Programming: How COVID-19 affects software developers and how their organizations can help. ArXiv200501127 Cs. (2020).

7. Klüppel, L.M., Pierce, L., Snyder, J.A.: Perspective-The Deep Historical Roots of Organization and Strategy: Traumatic Shocks, Culture, and Institutions. Organ. Sci. 29, 702-721 (2018). https://doi.org/10.1287/orsc.2017.1173.

8. Romano, N.C., Nunamaker, J.F.: Meeting analysis: Findings from research and practice. In: System Sciences, 2001. Proceedings of the 34th Annual Hawaii International Conference on. pp. 13-pp. IEEE (2001).

9. Waber, B.N.: Understanding the link between changes in social support and changes in outcomes with the sociometric badge, https://dspace.mit.edu/handle/1721.1/67762, (2011).

10. Whittaker, S., Frohlich, D., Daly-Jones, O.: Informal workplace communication: What is it like and how might we support it? In: Proceedings of the SIGCHI conference on Human factors in computing systems. pp. 131-137 (1994).

11. Nardi, B.A., Whittaker, S., Bradner, E.: Interaction and outeraction: instant messaging in action. In: Proceedings of the 2000 ACM conference on Computer supported cooperative work. pp. 79-88 (2000).

12. Daft, R.L., Lengel, R.H.: Organizational Information Requirements, Media Richness and Structural Design. Manag. Sci. 32, 554-571 (1986). https://doi.org/10.1287/mnsc.32.5.554.

13. Kleinbaum, A.M.: Reorganization and Tie Decay Choices. Manag. Sci. 64, 2219-2237 (2017). https://doi.org/10.1287/mnsc.2016.2705.

14. Bentley, T.A., Teo, S.T.T., McLeod, L., Tan, F., Bosua, R., Gloet, M.: The role of organisational support in teleworker wellbeing: A socio-technical systems approach. Appl. Ergon. 52, 207-215 (2016). https://doi.org/10.1016/j.apergo.2015.07.019.

15. Fonner, K.L., Roloff, M.E.: Why Teleworkers are More Satisfied with Their Jobs than are Office-Based Workers: When Less Contact is Beneficial. J. Appl. Commun. Res. (2010).

16. Lautsch, B.A., Kossek, E.E., Eaton, S.C.: Supervisory approaches and paradoxes in managing telecommuting implementation: Hum. Relat. (2009).

17. Ramey, G., Ramey, V.A.: The Rug Rat Race. National Bureau of Economic Research (2009). https://doi.org/10.3386/w15284.

18. Ciciolla, L., Luthar, S.S.: Invisible Household Labor and Ramifications for Adjustment: Mothers as Captains of Households. Sex Roles. 81, 467-486 (2019).

19. Chung, H.: Gender, Flexibility Stigma and the Perceived Negative Consequences of Flexible Working in the UK. Soc. Indic. Res. (2018). 
20. Barber, L.K., Santuzzi, A.M.: Please respond ASAP: Workplace telepressure and employee recovery. J. Occup. Health Psychol. 20, 172 (2015).

21. Nardi, B.A., Whittaker, S.: The place of face-to-face communication in distributed work. Distrib. Work. 83, 112 (2002).

22. Thompson, C.: What If Working From Home Goes on ... Forever?, https://www.nytimes.com/interactive/2020/06/09/magazine/remote-work-covid.html, (2020).

23. Tarafdar, M., Cooper, C.L., Stich, J.-F.: The technostress trifecta - techno eustress, techno distress and design: Theoretical directions and an agenda for research. Inf. Syst. J. 29, 6-42 (2019). https://doi.org/10.1111/isj.12169.

24. Kim, T.J., Bradbury, M.S., Olguin, D.O.: System and Method for Transforming Communication Metadata and Sensor Data into an Objective Measure of the Communication Distribution of an Organization, (2020).

25. Whittaker, S.: Theories and methods in mediated communication. (2003).

26. Beauregard, T.A., Basile, K.A., Canónico, E.: Telework: outcomes and facilitators for employees. In: Landers, R.N. (ed.) The Cambridge Handbook of Technology and Employee Behavior. pp. 511-543. Cambridge University Press, Cambridge, UK (2019).

27. Morgan, J., Goldsmith, M.: The Employee Experience Advantage: How to Win the War for Talent by Giving Employees the Workspaces they Want, the Tools they Need, and a Culture They Can Celebrate. Wiley, Hoboken, New Jersey (2017).

28. Oz, T.: Digital Trails of Work Stressors. Presented at the Social, Cultural, and Behavioral Modeling (2020). https://doi.org/10.31219/osf.io/wxcqp.

29. DeFilippis, E., Impink, S.M., Singell, M., Polzer, J.T., Sadun, R.: Collaborating During Coronavirus: The Impact of COVID-19 on the Nature of Work. National Bureau of Economic Research (2020). https://doi.org/10.3386/w27612.

30. Green, N.J.: A multi-level analysis of telework adoption and outcomes following a natural disaster: the experiences of two Christchurch organisations, (2014).

31. Podsakoff, P.M., MacKenzie, S.B., Lee, J.-Y., Podsakoff, N.P.: Common method biases in behavioral research: a critical review of the literature and recommended remedies. J. Appl. Psychol. 88, 879-903 (2003).

32. Wallace, A.F.C.: Human behavior in extreme situations; a study of the literature and suggestions for further research. Washington, NAS, National Research Council (1956).

33. Boell, S.K., Cecez-Kecmanovic, D., Campbell, J.: Telework paradoxes and practices: the importance of the nature of work. New Technol. Work Employ. 31, 114-131 (2016).

34. Leonardi, P.M., Treem, J.W., Jackson, M.H.: The connectivity paradox: Using technology to both decrease and increase perceptions of distance in distributed work arrangements. J. Appl. Commun. Res. 38, 85-105 (2010).

35. boyd, danah, Crawford, K.: Critical Questions for Big Data. Inf. Commun. Soc. (2012).

36. Cioffi-Revilla, C.: Bigger Computational Social Science: Data, Theories, Models, and Simulations -- Not Just Big Data. SSRN Electron. J. (2016).

37. Arkcoll, P.: Update: The Impact of COVID-19/WFH on Work Habits, Worklytics, https://www.worklytics.co/update-the-impact-of-covid-19-wfh-on-work-habits/.

38. Singer-Velush, N., Sherman, K., Anderson, E.: Microsoft Analyzed Data on Its Newly Remote Workforce, https://hbr.org/2020/07/microsoft-analyzed-data-on-its-newly-remoteworkforce, (2020). 